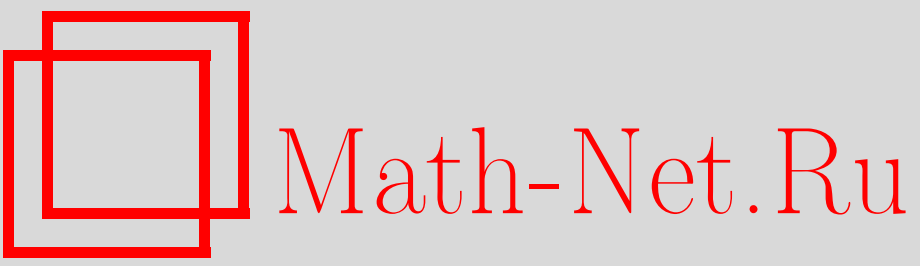

А. В. Гришин, О показателе представимости многообразия ассоциативных алгебр, УМН, 2002, том 57, выпуск 4, 169-170

DOI: https://doi.org/10.4213/rm538

Использование Общероссийского математического портала Math-Net.Ru подразумевает, что вы прочитали и согласны с пользовательским соглашением

http://www.mathnet.ru/rus/agreement

Параметры загрузки:

IP: 54.237 .206 .68

26 апреля 2023 г., 14:03:10 


\section{О ПОКАЗАТЕЛЕ ПРЕДСТАВИМОСТИ МНОГООБРАЗИЯ АССОЦИАТИВНЫХ АЛГЕБР}

\section{А. В. Гришин}

Пусть $k$ - бесконечное поле произвольной характеристики, $k_{r}$ - алгебра $(r \times r)$-матриц и $\mathfrak{M}$ многообразие ассоциативных алгебр надполем $k$. С каждым таким многообразием связаны такие числовые характеристики: сло жнность - натуральное число $r$, для которого $k_{r} \in \mathfrak{M}, k_{r+1} \notin \mathfrak{M}$, и кратность - индекс нильпотентности радикала относителшно свободной счетно-порожденной алгебры $\bar{F}$ этого многообразия. Для удобства все рассматриваемые алгебры предполагаются с единицей, а многообразия унитарно замкнутыми.

Наша цель - ввести еще один важный, как представляется, показатель многообразия $\mathfrak{M}$. Будем называть многообразие $\mathfrak{M}$ представимым, если его алгебра $\bar{F}$ вкладьвается в конечномерную алгебру над некоторым расширением поля $k$. Если $k$ - алгебраически замкнутое поле бесконечной степени трансцендентности над своим простым подполем, то представимость, как известно, равносильна равенству $\mathfrak{M}=\operatorname{Var}(A)$, где $A$ - некоторая конечномерная алгебра из $\mathfrak{M}$.

Пусть $\Omega$ - произвольное расширение поля $k$,

$$
F=k\left\langle x_{1}, \ldots, x_{i}, \ldots\right\rangle \text { и } \Omega F=\Omega k\left\langle x_{1}, \ldots, x_{i}, \ldots\right\rangle
$$

- свободные счетно-порожденные ассоциативные алгебры над полями $F$ и $\Omega$. Обозначим через $\mathfrak{M}^{\Omega}$ многообразие $\Omega$-алгебр, заданное тем же набором тождеств, что и $\mathfrak{M}$.

Всюду ниже сложность многообразия $\mathfrak{M}$ предполагается равной $r$. Положим

$$
L_{n}^{\Omega}=\underbrace{\Omega_{r} \oplus \cdots \oplus \Omega_{r}}_{n} \oplus \cdots \oplus \underbrace{\Omega \oplus \cdots \oplus \Omega}_{n}
$$

и рассмотрим свободные над $\Omega$ произведения

$$
L_{n}^{\Omega}\left\langle x_{1}, \ldots, x_{n}\right\rangle=L_{n}^{\Omega} * \Omega\left\langle x_{1}, \ldots, x_{i}, \ldots\right\rangle, \quad n=1,2, \ldots,
$$

т.е. алгебры обобщенных $L_{n}^{\Omega}$-многочленов от $n$ переменных. Рассмотрим, далее, фактор-алгебры

$$
R_{n}^{\Omega}=L_{n}^{\Omega}\left\langle x_{1}, \ldots, x_{n}\right\rangle /\left(x_{1}, \ldots, x_{n}\right)^{n},
$$

которые, очевидно, конечномерны и, в частности, $R_{1}^{\Omega}=L_{1}^{\Omega}=\Omega$. При $k=\Omega$ положим $R_{n}=R_{n}^{k}$. Пусть $S$ - произвольное множество многочленов из $F$ (например, $S-T$-идеал). Обозначим через $(S)_{n}^{\Omega}$ вербалшный идеал алгебры $R_{n}^{\Omega}$, порожденньй множеством $S$. Положим $R_{n}^{\Omega}(S)=R_{n}^{\Omega} /(S)_{n}^{\Omega}$, $R_{n}(S)=R_{n}^{k}(S)$. Ясно, что $R_{n}^{\Omega}(S)$ лежит в многообразии, заданном системой тождеств $S$. Из бесконечности поля $k$ прямо следует

Лемма. Если $I$ - T-идеал тождеств алгебры $R_{n}(S)$ в алгебре $F$, то $\Omega I-T$-идеал тождеств алгебры $R_{n}^{\Omega}(S)$ в алгебре $\Omega F$, причем $I_{1}=I_{2}$ тогда и только тогда, когда $\Omega I_{1}=\Omega I_{2}\left(\right.$ әде $I_{1}, I_{2}-T$-идеальц алгебры $\left.F\right)$.

Теорема. Следующие утверждения әквивалентны.

(1) Многообразие $\mathfrak{M}$, заданное системой $S$, представимо.

(2) Существует такое натуральное $n_{0}$, ито

$$
\operatorname{Var}\left(R_{n_{0}}(S)\right)=\operatorname{Var}\left(R_{n_{0}+1}(S)\right)=\cdots
$$

(3) Существует такое натуральное $n_{0}$, что $\mathfrak{M}=\operatorname{Var}\left(R_{n_{0}}(S)\right)$. 
НАБРОСОК ДОКАЗАТЕЛЬСТВА. Если многообразие $\mathfrak{M}$ - представимо, то в силу сказанного вьше для некоторого алгебраически замкнутого расширение $\Omega$ поля $k$ и для некоторой конечномерной $\Omega$-алгебры $A$ имеет место равенство $\mathfrak{M}^{\Omega}=\operatorname{Var}(A)$. Следовательно, для некоторого натурального $n_{0}$ существует сюръективный гомоморфизм алгебры $R_{n_{0}}^{\Omega}(S)$ на алгебру $A$. Тогда, очевидно,

В силу леммы

$$
\operatorname{Var}(A)=\operatorname{Var}\left(R_{n_{0}}^{\Omega}(S)\right)=\operatorname{Var}\left(R_{n_{0}+1}^{\Omega}(S)\right)=\cdots .
$$

$$
\operatorname{Var}\left(R_{n_{0}}(S)\right)=\operatorname{Var}\left(R_{n_{0}+1}(S)\right)=\cdots .
$$

Ясно, что алгебры $R_{n_{0}}(S), R_{n_{0}+1}(S), \ldots$ порождают многообразие $\mathfrak{M}$ (это следует из возможности аппроксимировать алгебру $\bar{F}$ конечномерньми алгебрами). Следовательно,

$$
\mathfrak{M}=\operatorname{Var}\left(R_{n_{0}}(S)\right)=\operatorname{Var}\left(R_{n_{0}+1}(S)\right)=\cdots
$$

Если $\mathfrak{M}=\operatorname{Var}(A)$, где $A$ - некоторая конечномерная $k$-алгебра, то $\bar{F}$ - алгебра общих элементов алгебры $A$, что и позволяет осуществить вложение алгебры $\bar{F}$ в конечномерную алгебру над некоторым расширением поля $k$.

СледСтвиЕ. В случае бесконечного поля представимость многообразия М равносильна равенству $\mathfrak{M}=\operatorname{Var}(A)$, где $A$ - некоторая конечномерная алгебра из $\mathfrak{M}$.

Наименьшее натуральное $n_{0}$ из приведенной выше теоремы назовем показателем представимости $\rho(S)=\rho(\mathfrak{M})$ многообразия $\mathfrak{M}$, а конечномерные алгебры $R_{n_{0}}(S), R_{n_{0}+1}(S), \ldots$ модельными алгебрами этого многообразия.

Ясно, что кратность многообразия $\mathfrak{M}$ не превосходит $\rho(S)$, что показывает важную роль показателя представимости данного многообразия. Зная показатель представимости, можно построить модельную алгебру многообразия. Однако само отыскание числа $\rho(S)$ - весьма трудная задача.

Из резултатов Левина [1] следует, что если $M_{r}$ - идеал тождеств алгебры $k_{r}$, то

$$
\rho\left(M_{r_{1}} \cdots M_{r_{l}}\right)=l .
$$

Если $c_{n}$ - многочлен Капелли порядка $n$, то при $n \geqslant 4$ в случае поля нулевой характеристики оценка показателя $\rho\left(c_{n}\right)$ - интересная и важная задача, решение которой еще впереди.

\section{СПИСОК ЛИТЕРАТУРЫ}

[1] J. Lewin // Trans. Amer. Math. Soc. 1974. V. 188. № 2. P. 293-317.

Московский педагогический

Принято редколлегией государственньй университет 